\title{
Electric field controlled transport of water in graphene nano-channels
}

\author{
Alper Tunga Celebi, ${ }^{1}$ Murat Barisik, ${ }^{2}$ and Ali Beskok ${ }^{1, a)}$ \\ ${ }^{1}$ Lyle School of Engineering, Southern Methodist University, Dallas, Texas 75205, USA \\ ${ }^{2}$ Department of Mechanical Engineering, Izmir Institute of Technology, Izmir, Turkey
}

(Received 14 July 2017; accepted 16 October 2017; published online 31 October 2017)

\begin{abstract}
Motivated by electrowetting-based flow control in nano-systems, water transport in graphene nanochannels is investigated as a function of the applied electric field. Molecular dynamics simulations are performed for deionized water confined in graphene nano-channels subjected to opposing surface charges, creating an electric field across the channel. Water molecules respond to the electric field by reorientation of their dipoles. Oxygen and hydrogen atoms in water face the anode and cathode, respectively, and hydrogen atoms get closer to the cathode compared to the oxygen atoms near the anode. These effects create asymmetric density distributions that increase with the applied electric field. Force-driven water flows under electric fields exhibit asymmetric velocity profiles and unequal slip lengths. Apparent viscosity of water increases and the slip length decreases with increased electric field, reducing the flow rate. Increasing the electric field above a threshold value freezes water at room temperature. Published by AIP Publishing. https://doi.org/10.1063/1.4996210
\end{abstract}

\section{INTRODUCTION}

Development of complex nano-fluidic systems with pumps, valves, and other flow control elements requires enhanced understandings of the structure, dynamics, and transport of nano-scale confined liquids under externally and locally applied fields. Previous experiences from microfluidic systems can help conceptualize nano-fluidic components. For example, electrowetting has been used in microfluidic systems to guide and mix droplets using locally applied electric fields. ${ }^{1,2}$ Controlling the surface wettability of nanochannels by locally applied electric fields can be used to regulate the flows. Graphene can be used as local electrodes and varying the electric charges on each electrode can generate the desired electric field. ${ }^{3,4}$ However such developments require an advanced understanding of nano-scale liquid transport phenomena, which can be achieved using atomistic simulations.

Liquid transport in nano-scale systems deviates from their micron and larger counterparts due to the scale and force-field effects. ${ }^{5}$ Scale effects become prominent when the molecular diameter becomes comparable to the dimensions of the flow conduit so that fluid molecules can no longer be considered as point particles. For example, the well-known density layering of liquids near the walls is a result of the liquid-wall force field interactions and the finite size of liquid molecules. ${ }^{6}$ These local effects confined to the near-wall region subside within several molecular diameters, and the fluid density reaches a constant bulk value away from the walls. ${ }^{7}$ The channel-averaged fluid density and apparent-viscosity increasingly deviate from their thermodynamic values with scale reduction. ${ }^{8}$ In addition, depending on the surface-liquid interaction strength, liquid

a)Author to whom correspondence should be addressed: abeskok@smu.edu. Tel.: 214-768-3200. Fax: 214-768-1473. may exhibit velocity slip, no-slip, or adsorption that greatly affect transport. ${ }^{5,9}$ However, these behaviors must be defined at the first liquid density layer rather than the walls since the liquid molecules cannot get closer to the walls more than a molecular diameter. For example, fluid slip-plane is at the first mobile density layer near the wall, which affects the apparent channel height. ${ }^{10}$ As a result of these effects, predictions of the continuum transport models become inaccurate with decreasing system dimensions, and eventually discrete transport of liquid molecules under the influence of wall force field effects dominates the flow. ${ }^{11}$

Molecular dynamics (MD) has been used to investigate electro-wetting behavior of nano-droplets on different surfaces. Zhang et al. ${ }^{12}$ reported enhanced wettability of water molecules on platinum surfaces with increased electric field strengths. Song et al. ${ }^{13}$ investigated spreading of nano-droplets under electric fields applied parallel to the solid surfaces and reported the decrease of the contact angle and formation of asymmetric droplet shapes. Giovambatistta et al. ${ }^{14}$ investigated the variation of liquid contact angle as a function of locally induced surface charges and showed a reduction in the contact angle with increased electrical charge density. A general trend in the simulations is the enhancement of surface wettability with increased electric fields, where the wetting angle decreases and the surface becomes more hydrophilic corresponding to stronger liquid-solid interactions. Polarizable water molecules reorient themselves in the electric field direction. ${ }^{15,16}$ Increasing the electric field magnitude aligns the dipole moments of the water molecules in the system and restricts their degree of freedom, which eventually results in electro-freezing, where the water molecules exhibit high-ordered crystalline structures. ${ }^{17,18}$ Svishchev and Kusalik $^{19}$ reported a study focusing on crystallization of liquid water on different types of surfaces under applied electric fields. Xia and Berkowitz ${ }^{20}$ investigated the structural behavior 
of water molecules confined between oppositely charged platinum walls. At sufficiently high electric fields, water molecules undergo a drastic change and form ice-like ordered structures.

To the best of our knowledge, there is no study investigating the effects of electric field on transport properties of nano-confined water such as viscosity, density, and slip length. In this study, non-equilibrium molecular dynamics (NEMD) simulations are performed for force-driven water flow through electrically charged graphene nano-channels. The main objective of this study is to elucidate the effect of electric field imposed by oppositely charged surfaces on the structural and transport properties of water confined in graphene nanochannels at a length scale, where the continuum behavior is still observed. We particularly focus on the behavior of deionized water and present results of density profiles, molecular orientations, velocity profiles, viscosities, and slip lengths after carefully fixing the thermodynamic state.

\section{THEORETICAL BACKGROUND}

Water transport in charged graphene nano-channels may exhibit variations in the slip length and viscosity. In order to assess such effects, we concentrate on force-driven flow between two parallel plates shown in Fig. 1 and review liquid transport using continuum fluid mechanics. The Navier-Stokes equations for steady, incompressible, fully developed, forcedriven Newtonian fluid flows can be simplified to result in the following equation:

$$
\frac{d^{2} u}{d z^{2}}=-\frac{f}{\mu}
$$

where $f$ is the driving body force, $\mu$ is the fluid viscosity, and $u(z)$ is the velocity field. At the liquid-solid interface, we assume Navier-type slip boundary condition given by

$$
u_{s}-u_{w}=\beta \frac{d u}{d z},
$$

where $u_{s}$ and $u_{w}$ are the liquid slip and wall velocities, respectively, and $\beta$ is the slip length. For generality of discussions, the slip length on each wall can be different. Using slip lengths

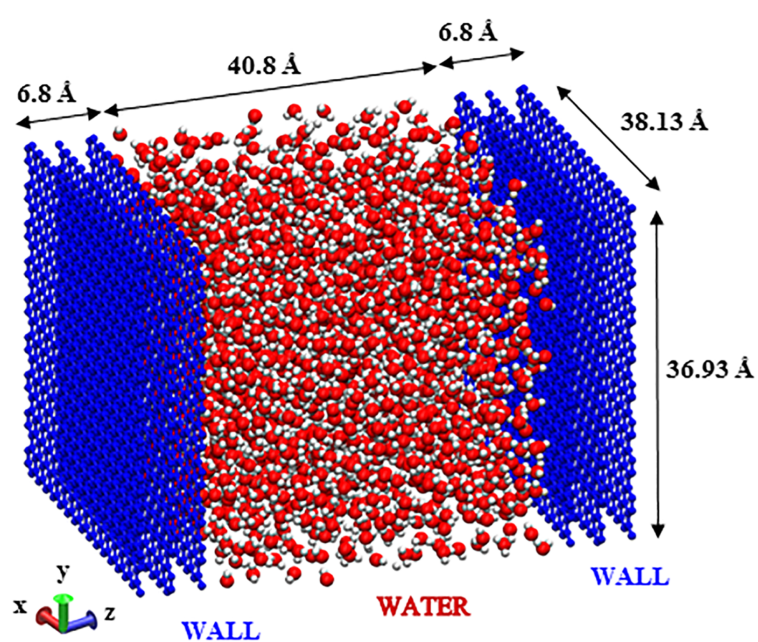

FIG. 1. Schematic and dimensions of the simulation domain. of $\beta_{L}$ on the left wall $(z=0)$ and $\beta_{R}$ on the right wall $(z=h)$, the velocity distribution in the channel is given by

$$
u(z)=\frac{f}{\mu}\left(-\frac{z^{2}}{2}+\frac{h\left(\beta_{R}+\frac{h}{2}\right)}{h+\beta_{L}+\beta_{R}} z+\beta_{L} \frac{h\left(\beta_{R}+\frac{h}{2}\right)}{h+\beta_{L}+\beta_{R}}\right) .
$$

For constant slip length $\beta$ on both surfaces, this equation reduces to following well-known form,

$$
u(z)=\frac{f h^{2}}{2 \mu}\left(-\left(\frac{z}{h}\right)^{2}+\left(\frac{z}{h}\right)+\left(\frac{\beta}{h}\right)\right) .
$$

In order to calculate the slip lengths and fluid viscosity, a curve fitting method using the velocity profiles obtained from MD simulations is utilized. ${ }^{8,21}$ First a parabolic velocity profile in the form of $u(z)=A z^{2}+B z+C$ is fitted to the MD simulation data, then the $A, B$, and $C$ coefficients are compared with the analytical solution given in Eq. (3). Accordingly, fluid viscosity is extracted using

$$
\mu=-\frac{f}{2 A} .
$$

This is followed by solving for the slip lengths of $\beta_{L}$ and $\beta_{R}$ analytically using the coefficients $B$ and $C$. The polynomialfit approach provides a good approximation when calculating viscosity and slip lengths from a parabolic velocity profile. However, curve fitting a parabolic profile to plug-like flows or velocity profiles with small parabolic components lead to inaccurate slip lengths or large statistical variations. ${ }^{22}$ For such cases, we used conservation of linear momentum in the flow direction, which provides equilibrium between the wall shear $\left(\tau_{w}\right)$ and total body force exerted on the water molecules as $\tau_{w}=\frac{f h}{2}$. Then, combining this equation with the constitutive law for Newtonian fluids as $\tau_{w}=\mu \frac{d u}{d y}$ and Navier-type slip equation given in Eq. (2), the slip length is calculated as

$$
\beta=\frac{2 \mu u_{s}}{f h}=\frac{2 \mu \bar{u}}{f h},
$$

where $u_{s}$ and $\bar{u}$ are the slip and average velocities, respectively. It is critical to state that we assumed slip velocity equal to the average velocity, which is the case for pure plug-flow behavior. Approach based on Eq. (6) results in accurate estimates of the interfacial slip behavior at the graphene-water interface. $^{23,24}$

\section{MOLECULAR DYNAMICS SIMULATION}

We carried out molecular dynamics simulations on a three-dimensional model system consisting of water molecules confined between graphitic solid walls. A schematic illustration of the simulation domain is shown in Fig. 1. Total dimensions of the domain in the lateral ( $\mathrm{x}$ and $\mathrm{y}$ ) and vertical ( $\mathrm{z}$ ) directions were set as $38.13,36.93$, and $54.4 \AA$, respectively. In the current study, the channel height is $h=40.8 \AA$. This dimension is specifically chosen so that both the continuum behavior and the graphene-water interface phenomenon are still observable. Therefore, the simulation domain is large enough to show density layering due to the wall force-field effect as well as a significant bulk region in the middle of the channel. For much narrower channels, the definition of the thermodynamic 
state and the derivation of continuum variables such as density, velocity, and viscosity become irrelevant, and water molecules exhibit discrete molecular transport as previously shown in the literature. $^{25-27}$

Each solid wall contains three defect-free graphene sheets separated by $3.4 \AA$ distance. Graphene wall layers in the walls were organized based on the Bernal (ABA) stacking arrangement on the XY plane. ${ }^{28}$ Wall atoms were constrained at their initial positions representing a cold wall behavior, while remaining particles in the system were free to move. Opposite but equal surface charges were taken into consideration on the left and right walls to satisfy the neutrality of the simulation box. Charged surfaces in an electrolyte solution act as electrodes that attract counter ions, creating an electrical double layer (EDL) to shield the surface charge. This phenomenon becomes dominant at high ionic concentration and substantially affects the structure and dynamics of water in nano-scale confinements. Wang et al. ${ }^{29}$ showed significant differences on the ion and liquid density distributions for a $\mathrm{LiClO} 4$-acetonitrile/graphite EDL capacitor at various surface charges. Unlike their study, we focus on the limit of deionized water and neglect any EDL formation on the electrodes.

Intermolecular (van der Waals and electrostatic) forces for all atomic species were described using Lennard-Jones (LJ) and long-range Coulombic potentials as follows:

$$
\varnothing\left(r_{i j}\right)=4 \varepsilon\left[\left(\frac{\sigma_{i j}}{r_{i j}}\right)^{12}-\left(\frac{\sigma_{i j}}{r_{i j}}\right)^{6}\right]+\frac{1}{4 \pi \varepsilon_{0}} \sum_{i}^{a} \sum_{j}^{b} \frac{q_{\mathrm{i}} q_{\mathrm{j}}}{r_{i j}}
$$

where $\varepsilon$ and $\sigma$ are the well-depth and molecular diameter, respectively. $\epsilon_{0}$ is the dielectric constant for vacuum, $q_{\mathrm{i}}$ values are the partial charges, and $r_{i j}$ is the distance between two atoms or charged sites.

Interactions between water molecules were calculated using a rigid four-site TIP4P/2005 model, which provides a good approximation to reproduce structural and hydrodynamic properties of liquid water in a wide range of temperatures. $^{30,31}$ For this water model, oxygen atoms do not carry partial charges. Instead, a massless negatively charged dummy atom is added along the bisector of the $\mathrm{H}-\mathrm{O}-\mathrm{H}$ bond angle. But, neutrality of a water molecule is satisfied by positively charged hydrogen atoms. Bond lengths and angles in water molecules were constrained by SHAKE algorithm in order to make the water molecules rigid. ${ }^{32}$ Only oxygen atoms were taken into considerations in our LJ calculations between water-water and water-graphene. Oxygen-carbon interactions were computed using an LJ potential accurately parameterized by experimental observation of water/graphene contact angle by Werder et al. ${ }^{33}$ Although covalent bonds between carbon atoms can be modeled using interatomic potentials such as adaptive intermolecular reactive bond order (AIREBO), ${ }^{34}$ we excluded these interactions due to the use of the cold-wall model, which eliminates all forces exerted on the carbon atoms. Keeping carbon atoms stationery does not substantially change the structure or dynamics of confined water, but it drastically reduces the computational cost. ${ }^{33,35}$ In addition, electrical charges are imposed on the carbon atoms at the water-graphene interface, while no charge is assigned to the rest. Coulombic interactions between all charged particles are taken into consideration. These long-range electrostatic interactions were handled by engaging particle-particle-particle mesh (P3M) method with a root-mean-accuracy of $10^{-5} \cdot 36$ PPPM maps atomic charges to a 3D mesh and enables 3D fast Fourier transform (FFT) to solve Poisson's equation on the mesh. Then, it interpolates electric fields on the mesh points back to the atoms. ${ }^{37}$ All short range LJ and Coulombic potentials were smoothly truncated at a cutoff distance of $1 \mathrm{~nm}$. Table I lists molecular parameters for all atomic pairs used in MD simulations. ${ }^{38}$

Large-Scale Atomic/Molecular Massively Parallel Simulator (LAMMPS) was employed in this study. ${ }^{39}$ Periodic boundary conditions were used in $\mathrm{x}$ - and $\mathrm{y}$-directions. A slab modification in the $\mathrm{z}$-direction was activated to calculate the electrostatic interaction for the reduced periodicity. ${ }^{40}$ This study simulates the well-known Poiseuille flow model between two electrically charged parallel plates to find the transport properties of nano-confined water. Before we applied any driving-force, we first thermally equilibrated each system in a canonical (NVT) ensemble. For this purpose, initial velocities on each water molecule were randomly assigned using a Gaussian distribution corresponding to the specified temperature. Initially, the MD system was run for 2 ns using 1 fs time steps for achieving an equilibrium state without any external forces. The thermodynamic state was fixed by maintaining the temperature at $300 \mathrm{~K}$ using the Nose-Hoover thermostat and keeping the bulk density of water away from the two graphitic surfaces at $997 \mathrm{~kg} / \mathrm{m}^{3}$. The temperature was calculated from the total kinetic energy, verifying the equilibrium state.

Starting from these equilibrium conditions, an external force was applied to conduct the flow simulations. For the flow cases, the Nose-Hoover thermostat was applied only to the degrees of freedom perpendicular to the flow direction. The flow was driven by a constant force in the y-direction applied to each atom of the water molecule with respect to their masses. The force for each surface charge density case was carefully chosen to generate velocities lower than $50 \mathrm{~m} / \mathrm{s}$. This is important to avoid any non-linear response due to high flow velocity and its dependence on the temperature. ${ }^{41,42}$ The linear response regime was verified by comparing the average channel velocities obtained by systematically increasing the driving force (not shown for brevity). The time scale for momentum diffusion was determined using $t_{d} \approx h^{2} / v$, where $v$ is the kinematic viscosity and $h$ is the channel height. ${ }^{10}$ We ensured reaching the steady state by initially running the system for $2 \mathrm{~ns}$, which corresponds to $12.5 t_{d}$. Afterwards we ran and time averaged the results for an additional 16 ns for data collection and statistical averaging, creating 1600 independent time-averaged data sets. In order to calculate the standard deviation and standard error, we used 20 independent

TABLE I. Molecular interaction parameters for atomic pairs.

\begin{tabular}{lccc}
\hline \hline Atom pair & $\sigma(\mathrm{nm})$ & $\varepsilon(\mathrm{kJ} / \mathrm{mol})$ & $\mathrm{q}(\mathrm{e})$ \\
\hline $\mathrm{H}-\mathrm{H}$ & 0 & 0 & 0.5564 \\
$\mathrm{O}-\mathrm{O}$ & 0.31589 & 0.7749 & -1.1128 (dummy) \\
$\mathrm{C}-\mathrm{O}$ & 0.3190 & 0.3921 & Varies \\
\hline \hline
\end{tabular}


samples obtained from the averaging of 80 consecutive data sets. The domain was divided into 1200 bins in the $\mathrm{z}$-direction to monitor the results. This number of slab-bins was determined so that the density distribution did not display any qualitative and quantitative differences, enabling proper resolution of the wall-liquid interface. ${ }^{43}$

\section{RESULTS}

In this section, we first examine the density profiles and molecular orientations obtained in MD simulations under zero and varying electric charges. This is followed by investigations of the velocity profiles, slip lengths, and viscosity variations for force-driven flows under applied electric charges.

In Fig. 2, we present the variation of density profiles for different surface charge densities $(\sigma)$. The surface charges are introduced as uniformly distributed partial charges to the carbon atoms at the graphite-water interface. Positive charges on the left wall represent anode, while negative charges on the right wall represent cathode. This configuration induces an electric field from the left wall to the right. Herein, surface charge densities of $0,6.56,13.12,19.68$, and 26.24 $\mu \mathrm{C} / \mathrm{cm}^{2}$ were taken into consideration, which are similar to the values used in previous MD studies. ${ }^{4,20}$ Resulting electric field strengths produced by these charges are $0,0.0925$, $0.185,0.2775$, and $0.37 \mathrm{~V} / \mathrm{nm}$, respectively, which are also in the range of electric field strengths used in earlier MD studies. $^{25,44,45}$ Although applying such high electric fields is difficult in experimental studies due to the dielectric breakdown of water, it is not totally impractical. ${ }^{46}$ For example, the pulse discharge method engages pulse voltages through two electrodes in an aqueous environment to generate an electric field on the order of $1 \mathrm{~V} / \mathrm{nm} .^{46-48}$

All density profiles present well-known layering phenomena due to the wall-liquid attraction and volume-exclusions as shown by Koplik and Banavar ${ }^{6}$ Three distinguishable density layering near each wall and a bulk region in the middle of the channel are observed. The bulk density is maintained at $997 \mathrm{~kg} / \mathrm{m}^{3}$ and the temperature is fixed at $300 \mathrm{~K}$, which fixes the thermodynamic state for all cases. Although the bulk densities are held constant, near-wall region exhibits

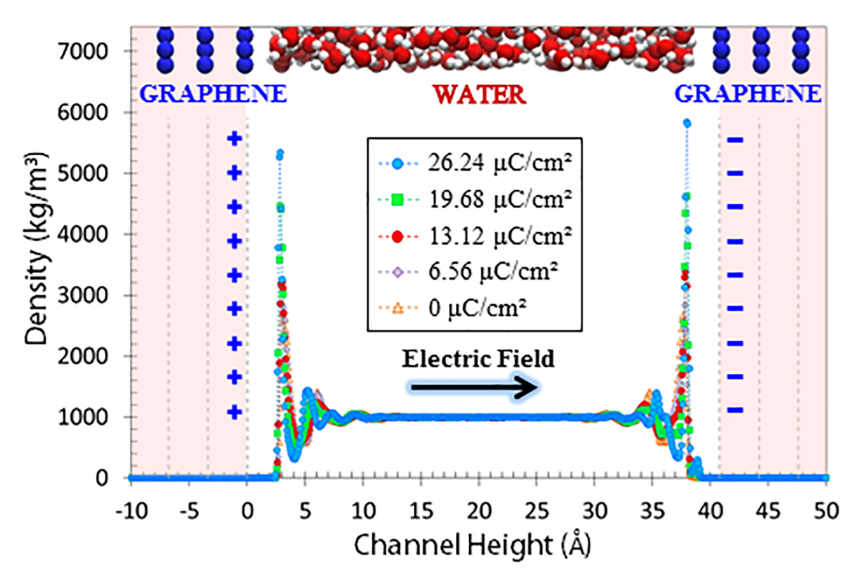

FIG. 2. Density distribution under different electric field strengths. different behavior for different surface charge densities. Dominated by increasing surface charges, wetting behavior of surfaces increases and locations and magnitudes of the density peaks differ.

In Fig. 3, we show the density profiles within $1 \mathrm{~nm}$ distance from left and right walls in order to better investigate the liquid-solid behavior at the interface. An increase on the surface charge increases the number of molecules at the first hydration layer due to higher interfacial energy and stronger wall-fluid interactions. Therefore, the magnitude of the first density peaks for each case increases with the surface charge. However, the second density peaks do not show any distinct trends. The third density peaks do not present any qualitative or quantitative differences for the given surface charges, because the effect of van der Waals forces from the walls almost diminishes at this location. In addition to the density magnitudes, locations of the density peaks move closer to the wall with increased surface charges. For example, the first density peak is $3.16 \AA$ away from the walls (defined at the center of the first graphene wall layer) in the absence of surface charges, while it is located $2.84 \AA$ away from the wall for the largest surface charge. Current results are in good agreement with Ref. 16. Density profiles in Figs. 3(a) and 3(b) are asymmetric under applied electric fields. One can notice a small density peak near the right wall in Fig. 3(b) caused by the hydrogen atoms pointing towards the negatively charged surface. Figures 3(c) and 3(d) show oxygen and hydrogen densities normalized by their average values. The density behavior mentioned above can be better understood by the normalized hydrogen densities, which displays an extra hydrogen density peak near the right wall. These peaks occur due to molecular orientations of water molecules, which change by increased electric field. Overall, we observe oxygen density peaks closer to the positive (left) wall, while hydrogen density peaks are closer to the negative (right) wall.

The density results show the influence of the electric field on the orientation of water molecules. To quantify molecular orientations, we computed the probability distribution of water molecules using prescribed angle calculations. An angle of $\theta$ is described between the wall surface normal vector and the dipole moment vector of a water molecule as illustrated in Fig. 4. ${ }^{49}$ Accordingly, the dipole vector of a water molecule points towards the surface when the angle is $180^{\circ}$, and it points away from the surface when the angle is $0^{\circ}$. Similar to the previous studies in the literature, ${ }^{50,51}$ only the water molecules in the first hydration shell (within $5 \AA$ distance from the wall) were taken into consideration as the interface region. Angle cosines and probability distribution curves of interfacial water with respect to the positively charged left wall are shown in Fig. 4. Probabilities are expected to change depending on the magnitude and direction of the applied electric field. In absence of surface charge, there is no distinct orientational preference, and the probability distribution shows nearly symmetric behavior. Under applied electric field, more water molecules rotate their dipole moments towards the electric field direction. In other words, the number of dipole moments pointing away from the left surface increases with increasing electric field. For the largest electric field, almost no water molecules are oriented towards the left surface. Two important factors determine the 

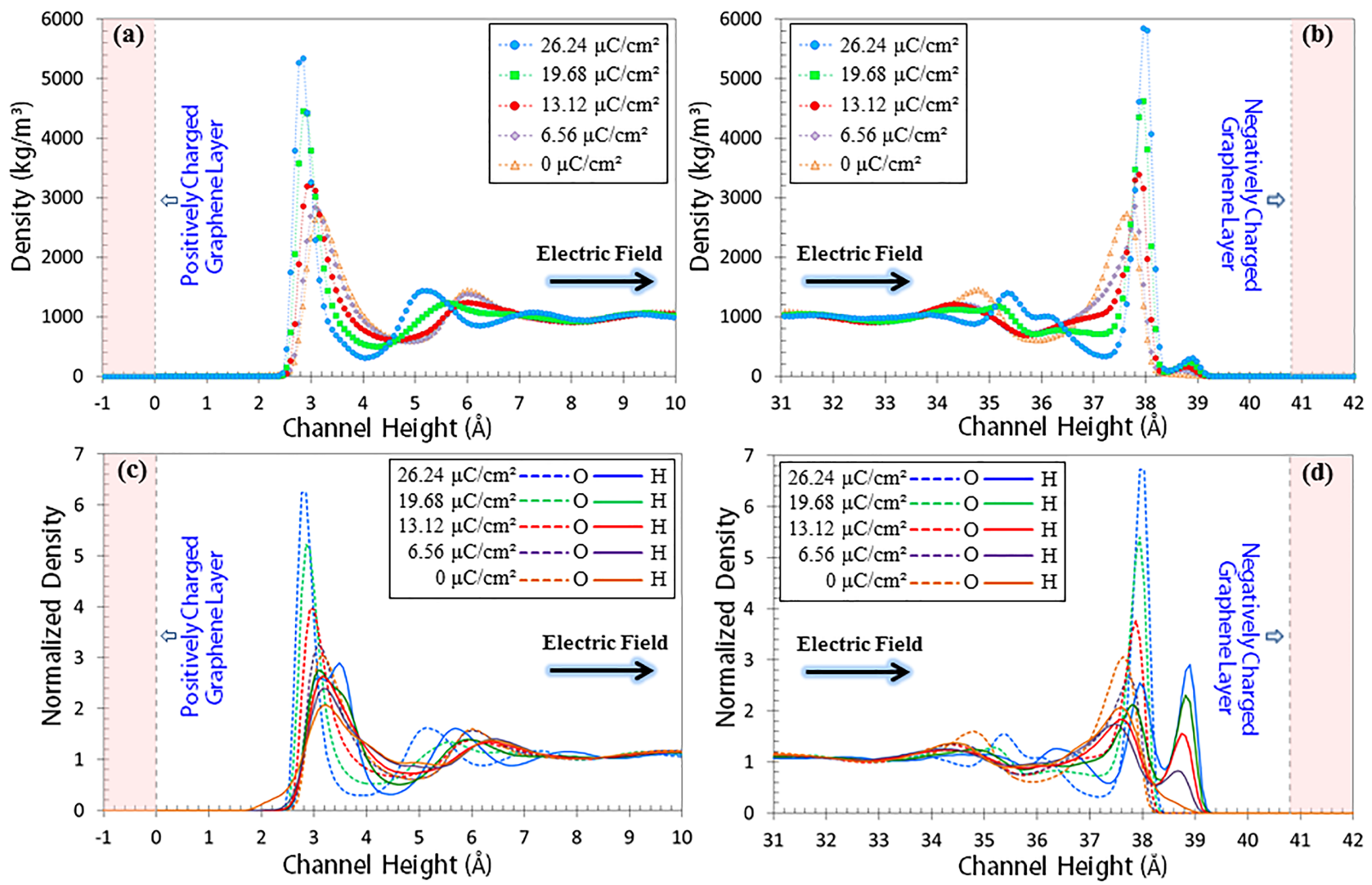

FIG. 3. Water density near positively charged left wall (a) and negatively charged right wall (b). Normalized oxygen and hydrogen densities near positively charged left wall (c) and negatively charged right wall (d).

molecular orientations here. First, the positive wall attracts negative oxygen atoms and repels positive hydrogen atoms through Coulombic interactions. Therefore, water molecules at the interface rotate towards the negatively charged right wall with increased electrostatic interactions. Second, the resulting electric field between charged surfaces produces additional force on each water molecule in the electric field direction, which dominates the orientation of water molecules through the entire channel. Probability distribution of water molecules at different $z$-locations in the channel exhibits very similar results to the orientations shown in Fig. 4.

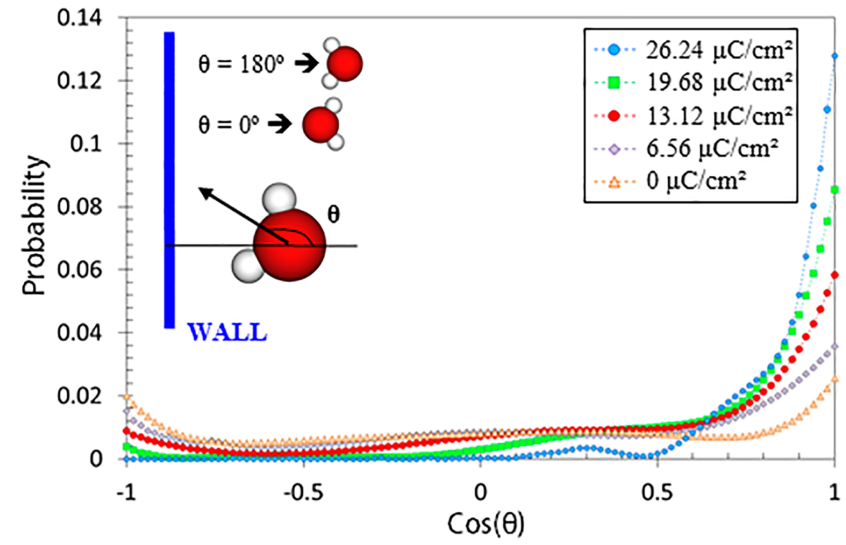

FIG. 4. Probability distribution of water molecules adjacent to the positively charged left wall.
Next, we present the results of the force-driven water flow between oppositely charged graphene nano-channels. Figure 5 shows the velocity profiles normalized with their average values for different surface charge densities. For zero surface charge, a plug-like velocity profile is obtained owing to the hydrophobic nature of graphene. This plug behavior originates from weak interfacial resistance at the water-graphene interface and results in large slip lengths. However, the velocity profiles assume different shapes with variation of the surface charges. With increased surface charge densities, the velocity profiles become parabolic with reduced slip lengths. This is

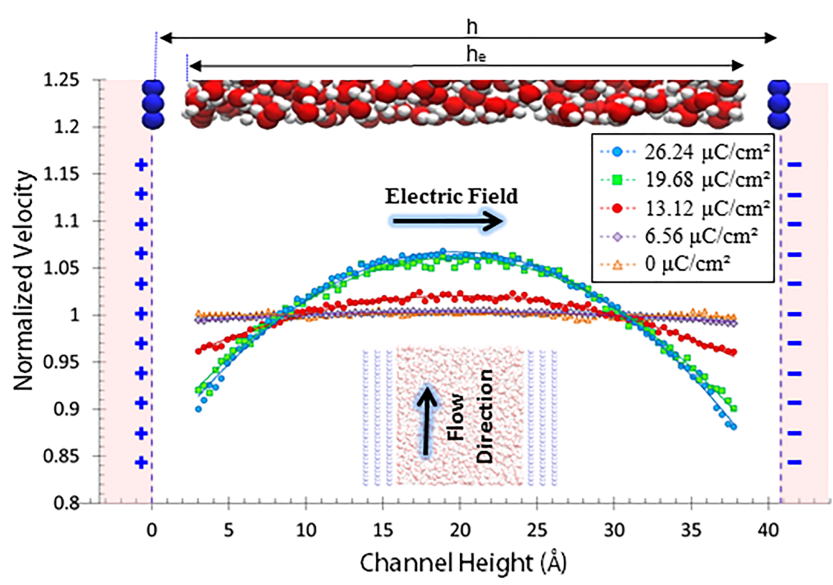

FIG. 5. Velocity profiles for different surface charges. 
due to the increasing liquid-wall coupling strength at the interface. Stronger wall-fluid interaction promotes the formation of parabolic velocity profiles. At surface charge magnitude of $6.56 \mu \mathrm{C} / \mathrm{cm}^{2}$, a very small parabolic component is observed on the velocity profile, and hence, this case still exhibits a plug-like velocity profile. However, distinguishable parabolic velocity profiles with reduced slip-lengths are formed above $13.12 \mu \mathrm{C} / \mathrm{cm}^{2}$. These results not only show control of the surface wetting behavior but also the alteration of nano-channel flow characteristics based on the applied electric field. Velocity profiles show asymmetry, which indicate that the slip length on the negatively charged right wall is lower than that on the positively charged left wall. This physical behavior can be explained by the density profiles in Fig. 3, where hydrogen atoms get closer to the negatively charged left wall, which induce stronger coupling at the interface and reduced velocity slip, compared with the left wall.

In Fig. 6, we show variations in viscosity and slip length under different surface charge densities. It is crucial to understand how to actively control transport properties with variation of electric field and at the same time, assess deviation of the results from continuum predictions. Viscosity and slip lengths in this study were calculated comparing the streaming velocity profiles obtained from NEMD simulations with continuum flow models such as Poiseuille flow (see Sec. II). One can also calculate these transport properties using GreenKubo relations in equilibrium MD (EMD) simulations, which integrates time correlation functions at equilibrium state. ${ }^{52}$ Using Green-Kubo relations, shear viscosities of different water models were calculated in several studies in the literature. ${ }^{53-55}$ They found the viscosity of the TIP4P/2005 model in the range of $820-855 \mu \mathrm{Pa}$ s which is in good agreement with the
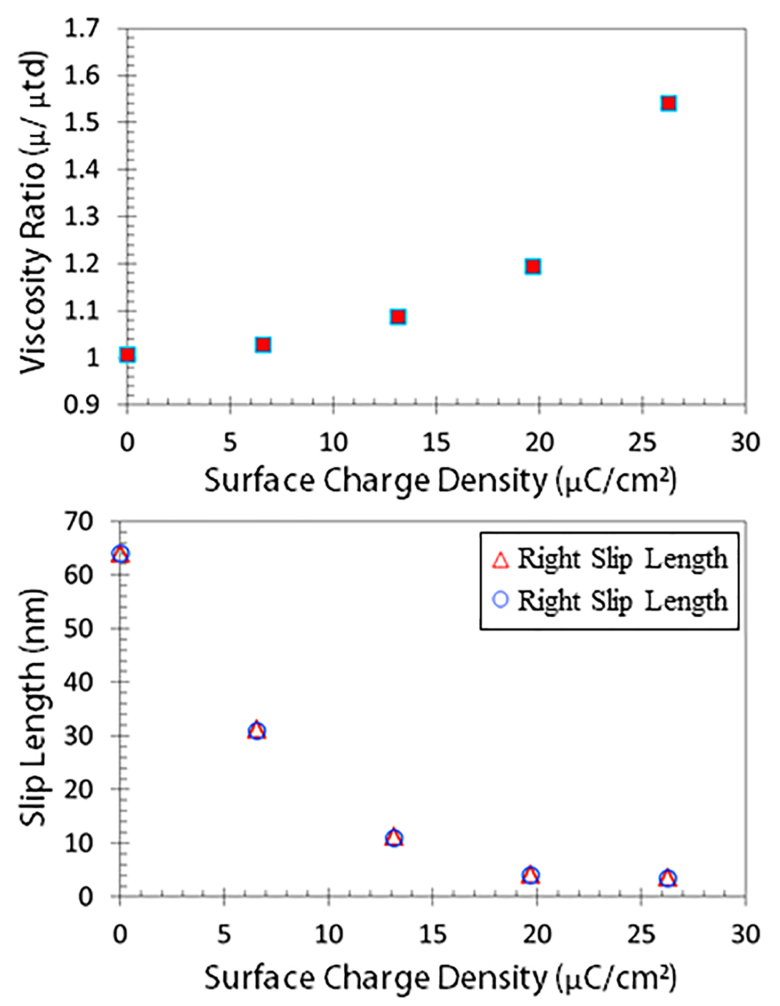

FIG. 6. Viscosity ratios and slip length variations with the surface charge. thermodynamic viscosity $\left(\mu_{t d}=858 \mu \mathrm{Pa} \mathrm{s}\right)$ of water at $300 \mathrm{~K}$ and $997 \mathrm{~kg} / \mathrm{m}^{3}$. For zero surface charge, we utilized this thermodynamic viscosity to predict the slip length using Eq. (6). Our previous studies suggest that the continuum predictions on the properties of bulk water at known thermodynamic state still hold in nanoscale up to channel heights of 2-3 nm. ${ }^{8,10}$ The velocity profile shows plug-like behavior on the neutral graphene surface with a very large slip. Applying polynomial fit method to this velocity profile produces large statistical uncertainties in the calculation of the slip length as previously discussed in the study by Kannam et al. ${ }^{22}$ Here, we used an alternative approach similar to Falk et al., ${ }^{24}$ where we used Eq. (6) assuming that the slip velocity is equal to the average velocity and predicted slip length at the first water density peak as $64 \mathrm{~nm}$. This value is in good agreement with the values reported in the literature. Kannam et $a l .{ }^{22}$ estimated the slip length of water on a planar graphene surface as $60 \pm 6 \mathrm{~nm}$ using EMD simulations. Xiong et al. ${ }^{56}$ applied the GreenKubo relation and calculated the slip length approximately as $54 \mathrm{~nm}$. Koumoutsakos et al. ${ }^{57}$ calculated the water/graphene slip length as large as $63 \mathrm{~nm}$ by Couette flow MD calculations. Variations in the slip lengths reported in the literature could be a result of different potential parameters used in water-water and graphene-water interactions and also the location of the slip plane. Based on our previous studies, we define the slip plane at the first water density peak near the surface. ${ }^{10}$ Slip lengths on the wall $\left(\beta_{W}\right)$ and the slip plane $\left(\beta_{S P}\right)$ are related to each other by $\beta_{S P}=\beta_{W}+L_{O}$, where $L_{O}$ is the distance between the wall plane and the location of the first density peak. In this study, we present all slip lengths at the slip plane (i.e., $\beta=\beta_{S P}$ ) and $L_{O}=0.31 \mathrm{~nm}$.

For surface charges equal to $13.12 \mu \mathrm{C} / \mathrm{cm}^{2}$ or larger, we calculated the viscosities and the slip lengths using the polynomial fit approach. For $6.56 \mu \mathrm{C} / \mathrm{cm}^{2}$ surface charge, there is a weak parabolic behavior, while the velocity profile is mostly plug-like. We used both polynomial fit and pluglike methods together to determine the viscosity and the slip lengths. Figure 6 shows a nonlinear increase in fluid viscosity with increased surface charge and electric field. Absolute viscosity of $1330 \mu \mathrm{Pa} \mathrm{s}$ is obtained for the largest surface charge density, while $\mu=863 \mu \mathrm{Pa}$ s for uncharged surfaces. Figure 6 also shows decreasing slip-lengths with increased surface charge for each wall. The slip length for the uncharged water-graphene surface is $64 \mathrm{~nm}$, and it decreases to values lower than $4 \mathrm{~nm}$ for the largest surface charge density case. Combined effects of increased viscosity and decreased slip for the largest surface charge density case exhibit 20 times reduction in the volumetric flow rate between the two cases.

Table II shows the viscosity, left and right slip lengths as well as the applied force per molecule. Slip length magnitudes on the left and right walls exhibit small but non-ignorable differences. As the electric field is increased, asymmetry in the slip lengths becomes more pronounced. This is mainly because of the increased differences in water density between the two walls. More water molecules assemble near the right wall due to the electric field and rotate their dipoles towards the right wall creating a small hydrogen density peak [See Fig. 3(b)] that affects the slip velocity. Consequently, the slip lengths on 
TABLE II. Viscosity and slip-length results for different surface charges.

\begin{tabular}{lcccccl}
\hline \hline $\begin{array}{l}\text { Surface charge } \\
\left(\mu \mathrm{C} / \mathrm{cm}^{2}\right)\end{array}$ & $\begin{array}{c}\text { Viscosity } \\
(\mu \mathrm{Pa} \mathrm{s})\end{array}$ & $\begin{array}{c}\text { Left slip } \\
\text { length }(\mathrm{nm})\end{array}$ & $\begin{array}{c}\text { Right slip } \\
\text { length }(\mathrm{nm})\end{array}$ & $\begin{array}{c}\text { Normalized shift } \\
\text { distance }\end{array}$ & $\begin{array}{c}\text { Driving force } \\
(\mathrm{N} / \text { molecule })\end{array}$ & p-value \\
\hline 26.24 & 1330.3 & $3.91 \pm 0.05$ & $3.46 \pm 0.06$ & 0.019 & $1.92 \times 10^{-13}$ & 0.0001 \\
19.68 & 1028.9 & $4.54 \pm 0.09$ & $4.11 \pm 0.09$ & 0.017 & $1.04 \times 10^{-13}$ & 0.003 \\
13.12 & 937.5 & $11.7 \pm 0.2$ & $11.1 \pm 0.2$ & 0.013 & $6.09 \times 10^{-14}$ & 0.09 \\
6.56 & 890.4 & $31.8 \pm 0.5$ & $31.0 \pm 0.5$ & 0.010 & $2.08 \times 10^{-14}$ & 0.5 \\
0 & 863.4 & $64.1 \pm 0.6$ & $64.1 \pm 0.6$ & $\ldots$ & $1.44 \times 10^{-14}$ & $\cdots$ \\
\hline \hline
\end{tabular}

the positively charged left wall are larger than the negatively charged right wall. Figure 7 shows MD-calculated velocity distribution at $\sigma=26.24 \mu \mathrm{C} / \mathrm{cm}^{2}$ with asymmetric curve fit to MD data using Eq. (3). We also present a symmetric parabolic velocity fit using constant slip length $\left(\beta_{A v e}\right)$ as an average of the right and left wall slip lengths and Eq. (4). MD-based velocity profile is clearly asymmetric, overshooting the symmetric velocity profile on left half of the domain and undershooting it on the right half of the domain. Furthermore, the symmetry axis of the MD velocity profile is shifted towards the left wall by a distance $l$. In Table II, we present the location of maximum velocity magnitudes measured from the channel center as the normalized shift distance $l / h$ towards the cathode. This shift distance decreases with reduced surface charge. In order to prove that the reported asymmetries are due to different slip behaviors on the left and right walls, we also provide statistical uncertainties in the reported slip lengths in Table II. Standard error $(S E)$ in the left and right slip lengths is calculated using the slip lengths obtained from asymmetric velocity fits made to 20 independent samples $(n)$ by $S E=S / \sqrt{n}$, where $S$ is the standard deviation. Each independent sample was obtained from the averaging of 80 consecutive time-averaged data sets. As can be seen, $S E$ is below the differences in the reported left and right wall slip lengths. In Table II, we also present the p-values obtained using student's t-test to compare the slip lengths on the positively and negatively charged surfaces. In the Student's t-test, p-values lower than 0.05 indicate significant difference. ${ }^{58}$ Accordingly, the reported slip lengths for $\sigma=26.24$ and $\sigma=19.68 \mu \mathrm{C} / \mathrm{cm}^{2}$ cases are statistically different. It is important to note that simulations conducted in

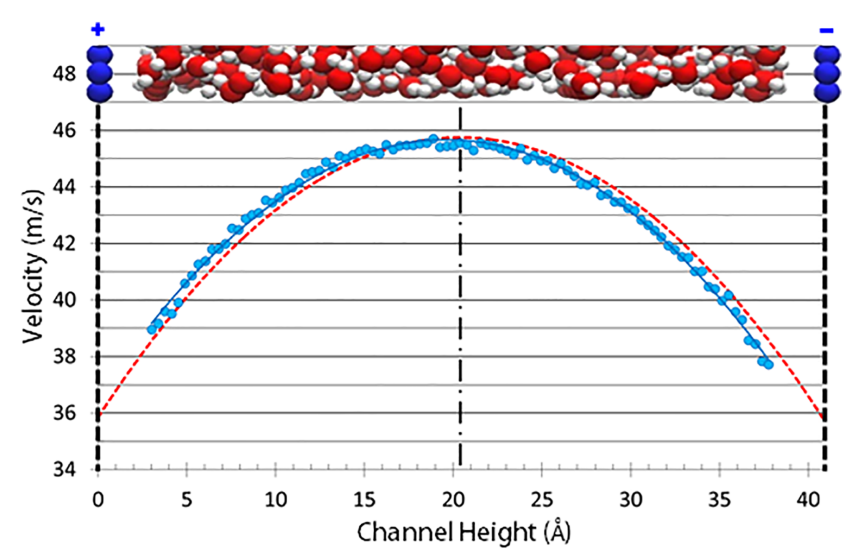

FIG. 7. Velocity distributions at $\sigma=26.24 \mu \mathrm{C} / \mathrm{cm}^{2}$. Blue dots are MD data, blue line is asymmetric velocity fit to MD data, red line is the symmetric parabolic fit with $\beta_{A v e}$, and the channel center is shown with black dashed line. $h=68 \AA$ channel at surface charge density of $26.24 \mu \mathrm{C} / \mathrm{cm}^{2}$ resulted in nearly identical density profiles, viscosity, and slip lengths, verifying that the presented transport phenomenon is scale independent for large enough channels that maintain continuum behavior (results not shown for brevity).

So far, we investigated the variation of the structure and dynamics of water due to the resulting electric field by oppositely charged surfaces with surface charges smaller than 30 $\mu \mathrm{C} / \mathrm{cm}^{2}$. As the surface charge is increased to a value more than $30.81 \mu \mathrm{C} / \mathrm{cm}^{2}$, density profiles experience a drastic change. For such a case, the constant bulk region in the center of the channel is replaced by a regular density layering, which implies that water transitions to a highly ordered crystalline structure. This threshold value was determined by systematically increasing the surface charge so that there is no more constant bulk density in the middle of the channel. Figure 8(a) shows normalized hydrogen and oxygen density profiles and the normalized velocity distribution for a surface charge of 32.78 $\mu \mathrm{C} / \mathrm{cm}^{2}$. Unlike the previous cases, a distinguishable density layering dominates the bulk region, while density peaks in the near-wall region are still observed due to strong wall force-field effects. These two behaviors elucidate the distinct alignment in the center of the channel forming the crystallized phase but still liquid water remains in the near-wall region, as observed in Fig. 8(b). Due to crystallization of water, the velocity profile is plug-like in the bulk of the channel. Sharp velocity gradients are observed in the near-wall region due to the presence of liquid water near the walls. This crystalline phase arranges water molecules in a hexagonal configuration displaying the characteristics of a solid state like hexagonal ice in the center of the channel. ${ }^{20,59}$ In addition, the nonlinear increase in the viscosity diverges to infinity, which supports solidification (see Fig. 6). This phenomenon, known as electro-freezing, was previously reported in multiple computational ${ }^{17,18,20}$ and experimental $^{60,61}$ studies in the literature. If the crystallization is thermodynamically and kinetically favorable, a stable heterogeneous nucleation suddenly appears on a solid substrate when the activation energy (free-energy barrier) is overcome. ${ }^{18,61,62}$ Therefore water can crystallize even at room temperature. ${ }^{63} \mathrm{~A}$ strong electric field facilitates ice nucleation by increasing the rate of formation of stable nuclei. An electric field aligns the dipoles of water molecules and restricts their degree of freedom normal to the resulting electric field, where the entropy of the liquid phase substantially drops. ${ }^{17}$ If the electric field is strong enough, the amount of reduced entropy closes the entropy difference between liquid and solid state leading to formation of stable, crystalline ice-like structures. ${ }^{17,64}$ It should be noted that freezing of water in nano-confinements also 
(a)

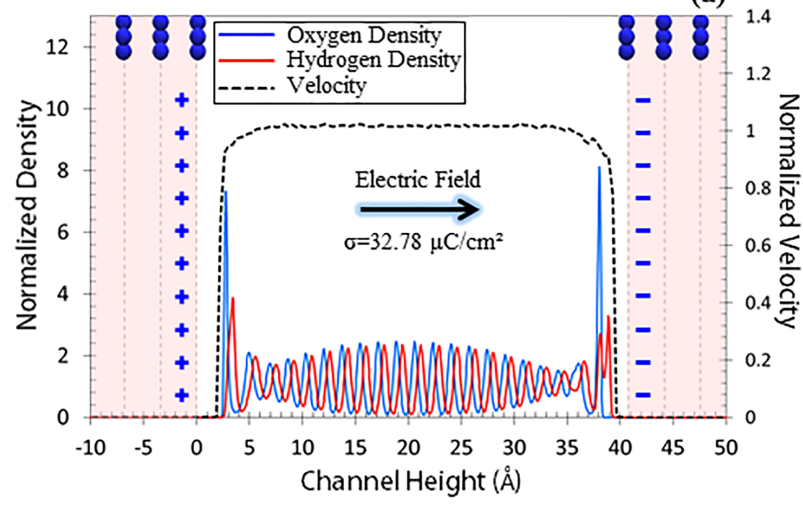

(b)

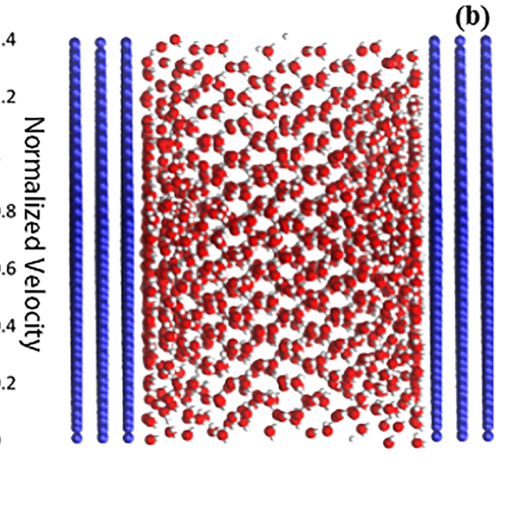

FIG. 8. Crystallization of water molecules. (a) Normalized hydrogen and oxygen density and velocity profiles; (b) Schematic representation from MD simulations. depends on several other variables such as model size ${ }^{65}$ surface geometry and chemistry, ${ }^{19}$ water model,${ }^{66}$ temperature, ${ }^{66}$ and applied electric field strength. Yan and Patey ${ }^{65}$ showed that ice nucleation and growth can only proceed when a certain size threshold is overcome. In addition, crystallization time and geometry shows significant variations on different crystallographic planes in different model sizes. ${ }^{19,65}$ This study only investigates the effect of the electric field strength on the estimation of electro-freezing with a fixed model size and geometry.

From Fig. 8, we can conclude that electric field is not strong enough to produce a perfect complete crystalline structure through the entire channel. Therefore, we applied an extreme electric field using a surface charge density of 98.40 $\mu \mathrm{C} / \mathrm{cm}^{2}$. Figure 9 illustrates the fully crystallized simulation domain and multiple views from different angles to clarify ice formation. In this case, a perfect hexagonal configuration of water molecules dominates the channel height, except in the near-wall region. Density distribution at the interface is mainly originated from local pressure buildup due to the constant simulation volume. The density of water reduces during freezing and ice density becomes approximately $920 \mathrm{~kg} / \mathrm{m}^{3}$ after the phase transition. ${ }^{67}$ However, constant volume and total number of molecules due to the used NVT ensemble prevent uniformly achieving this density value. Dominated by strong intermolecular forces between the wall and water molecules, the surplus molecules assemble near the walls and increase local pressure. This results in a liquid-like distribution at the interface. Addressing this issue, we calculated the density of the domain by excluding the irregular water molecule aggregation in the near-wall region. We found the density as $917 \mathrm{~kg} / \mathrm{m}^{3}$, which is in good agreement with the density of hexagonal ice at $0{ }^{\circ} \mathrm{C}$. $^{68}$

This solid-like ordering is an overall result of the alterations in hydrogen bonding networks due to the electric field. In liquid form, water molecules move randomly through the space, constantly breaking and reforming hydrogen bonds between the molecules. ${ }^{69}$ With very strong electric field, the orientation of the molecules drastically changes, resulting in fully aligned dipole moments through the electric field direction as shown in Fig. 9. Consequently, the random motion of liquid water diminishes, and the molecules become relatively locked at their positions. For such cases, hydrogen bonds form more frequently without breaking, creating a stable and energetically more favorable regular pattern. In reality, a water molecule in ice hydrogen bonds with four other neighboring molecules to create a tetrahedral crystal lattice, while the number of hydrogen bonds per molecule in the liquid phase is lower than this value. ${ }^{69} \mathrm{We}$ analyzed the liquid water and resultant solid-like phase by quantifying the number of hydrogen

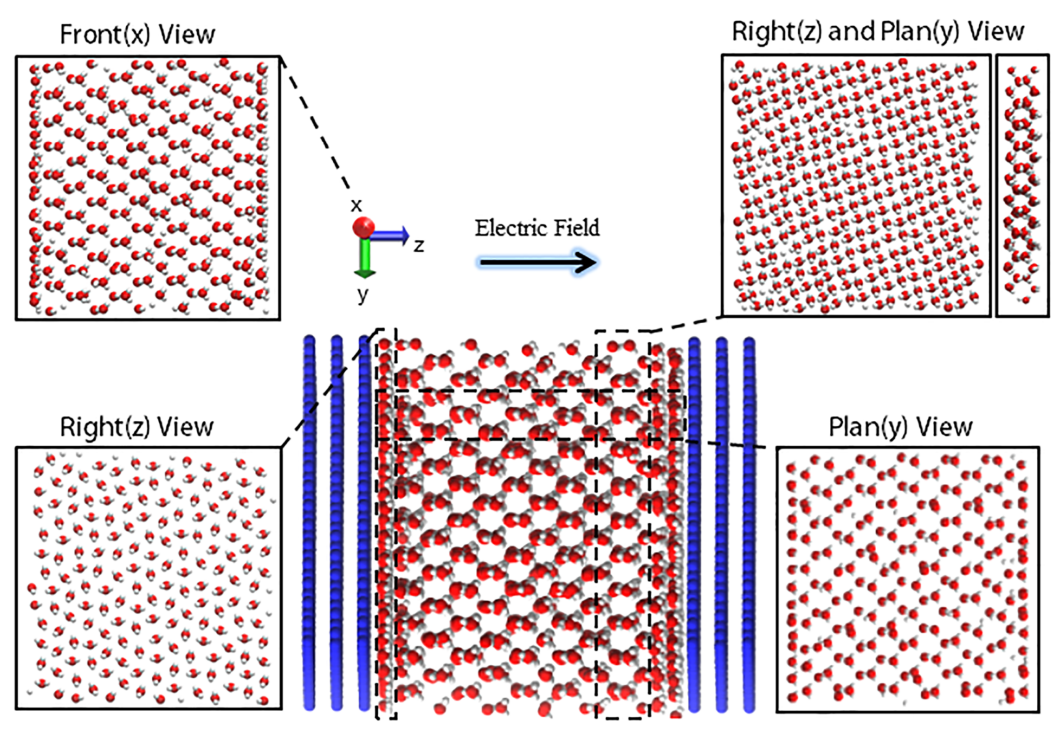

FIG. 9. Fully crystallized water domain. 
bonds per molecule in the bulk region of the channel following the geometric criteria in the study by Martí. ${ }^{70}$ For the uncharged case, the average number of hydrogen bonds per water molecule in the bulk region was 3.41, which reasonably agrees with the experimental value of 3.3 reported by Smith et al. ${ }^{71}$ and numerical result of 3.50 by Ho and Striolo. ${ }^{50}$ This value increases with an increased electric field in the system. Ritos et al. ${ }^{72}$ also showed that electric field increased the average number of hydrogen bonds per water molecule in a carbon nanotube from 3.50 to 3.95 , where they pointed out solidification at large field strengths. We calculated the average number of hydrogen bonds in the fully crystallized region in Fig. 9 as 3.97 , which is similar to ice. ${ }^{73}$

\section{CONCLUSIONS}

Using MD simulations, we investigated force-driven water flow in graphene nano-channels with opposing surface charges. Varying the surface charge density changes the applied electric field, which significantly alters the surface wetting and flow characteristics. With the increased electric field, the first water-density peaks get closer to the electrodes and the magnitudes of the density peaks increase; and asymmetric density distributions are observed due to water molecules orienting their dipoles towards the electric field direction. Force-driven flows under applied electric fields exhibit increased water viscosity and decreased slip lengths. For example, the slip-length of water on graphene surfaces at $26.24 \mu \mathrm{C} / \mathrm{cm}^{2}$ charge density is 16 times smaller than that on electrically neutral surfaces, and the water viscosity increases nearly $54 \%$ from its thermodynamic value. Furthermore, asymmetric velocity profiles are observed with increased electric fields, resulting in smaller slip lengths on the cathodes than the anodes. This is due to positively charged hydrogen molecules getting closer to the cathode compared to the position of negatively charged oxygen molecules near the anode. Above $31 \mu \mathrm{C} / \mathrm{cm}^{2}$ charge density, electro-freezing is observed, where water crystallizes in hexagonal configuration in the middle of the channel and liquid water is observed near the electrodes. Force-driven flow of this system shows transition from parabolic velocity profile to plug-like motion of ice with large velocity gradients near the walls. Further increases in the electric field enables better transition to the solid state by providing more populated hexagonal configuration of water molecules. Overall, results show the possibility of flow control using charged graphitic surfaces, where applied electric fields can substantially decrease the flow rate by reducing the slip length and increasing the water viscosity, eventually stopping the flow due to ice formation. These findings are also relevant in further miniaturization of electrowetting based droplet microfluidic systems, where slip length differences on the anodes and cathodes may lead to variations in the droplet wetting angle on the electrode surfaces.

\footnotetext{
${ }^{1}$ H. Moon, S. K. Cho, R. L. Garrell, and C. J. C. Kim, J. Appl. Phys. 92, 4080 (2002).

${ }^{2}$ F. Mugele and J. C. Baret, J. Phys.: Condens. Matter 17, R705 (2005).

${ }^{3}$ G. Jo, M. Choe, S. Lee, W. Park, Y. H. Kahng, and T. Lee, Nanotechnology 23, 112001 (2012).

${ }^{4}$ R. K. Kalluri, D. Konatham, and A. Striolo, J. Phys. Chem. C 115, 13786 (2011).
}

${ }^{5}$ G. Karniadakis, A. Beskok, and N. Aluru, Simple Fluids in Nanochannels (Springer, 2005).

${ }^{6}$ J. Koplik and J. R. Banavar, Annu. Rev. Fluid Mech. 27, 257 (1995).

${ }^{7}$ J. Lyklema, Fundamentals of Interface and Colloid Science: Soft Colloids (Academic Press, 2005), Vol. 5.

${ }^{8}$ J. Ghorbanian and A. Beskok, Microfluid. Nanofluid. 20, 121 (2016).

${ }^{9}$ A. Koklu, J. Li, S. Sengor, and A. Beskok, Microfluid. Nanofluid. 21, 124 (2017).

${ }^{10}$ J. Ghorbanian, A. T. Celebi, and A. Beskok, J. Chem. Phys. 145, 184109 (2016).

${ }^{11}$ S. Joseph and N. Aluru, Nano Lett. 8, 452 (2008).

${ }^{12}$ J. Zhang, M. K. Borg, K. Ritos, and J. M. Reese, Langmuir 32, 1542 (2016).

${ }^{13}$ F. Song, B. Li, and C. Liu, Langmuir 29, 4266 (2013).

${ }^{14}$ N. Giovambattista, P. G. Debenedetti, and P. J. Rossky, J. Phys. Chem. B 111, 9581 (2007).

${ }^{15}$ C. D. Daub, D. Bratko, K. Leung, and A. Luzar, J. Phys. Chem. C 111, 505 (2007).

${ }^{16}$ T. A. Ho and A. Striolo, J. Chem. Phys. 138, 054117 (2013).

${ }^{17}$ R. Zangi and A. E. Mark, J. Chem. Phys. 120, 7123 (2004).

${ }^{18}$ J. Yan and G. Patey, J. Phys. Chem. Lett. 2, 2555 (2011).

${ }^{19}$ I. M. Svishchev and P. G. Kusalik, Phys. Rev. Lett. 73, 975 (1994).

${ }^{20}$ X. Xia and M. L. Berkowitz, Phys. Rev. Lett. 74, 3193 (1995).

${ }^{21}$ J. Backer, C. Lowe, H. Hoefsloot, and P. Iedema, J. Chem. Phys. 122, 154503 (2005).

${ }^{22}$ S. Kumar Kannam, B. Todd, J. S. Hansen, and P. J. Daivis, J. Chem. Phys. 136, 024705 (2012).

${ }^{23}$ S. K. Kannam, B. Todd, J. S. Hansen, and P. J. Daivis, J. Chem. Phys. 135, 144701 (2011).

${ }^{24}$ K. Falk, F. Sedlmeier, L. Joly, R. R. Netz, and L. Bocquet, Nano Lett. 10, 4067 (2010).

${ }^{25}$ R. Qiao and N. Aluru, J. Chem. Phys. 118, 4692 (2003).

${ }^{26}$ K. P. Travis, B. Todd, and D. J. Evans, Phys. Rev. E 55, 4288 (1997).

${ }^{27}$ J. A. Thomas and A. J. McGaughey, Nano Lett. 8, 2788 (2008).

${ }^{28}$ A. Yacoby, Nat. Phys. 7, 925 (2011).

${ }^{29}$ Z. Wang, Y. Yang, D. L. Olmsted, M. Asta, and B. B. Laird, J. Chem. Phys. 141, 184102 (2014).

${ }^{30}$ L. Liu and G. Patey, J. Chem. Phys. 141, 18C518 (2014).

${ }^{31}$ J. L. Abascal and C. Vega, J. Chem. Phys. 123, 234505 (2005).

${ }^{32}$ S. Miyamoto and P. A. Kollman, J. Comput. Chem. 13, 952 (1992).

${ }^{33}$ T. Werder, J. H. Walther, R. Jaffe, T. Halicioglu, and P. Koumoutsakos, J. Phys. Chem. B 107, 1345 (2003).

${ }^{34}$ A. T. Celebi, M. Kirca, C. Baykasoglu, A. Mugan, and A. C. To, Comput. Mater. Sci. 88, 14 (2014).

${ }^{35}$ J. A. Thomas, A. J. McGaughey, and O. Kuter-Arnebeck, Int. J. Therm. Sci. 49, 281 (2010).

${ }^{36}$ S. Plimpton, R. Pollock, and M. Stevens, "Particle-mesh Ewald and rRESPA for parallel molecular dynamics simulations," in Proc. of the Eighth SIAM Conference on Parallel Processing for Scientific Computing, 1997, pp. $8 \mathrm{e} 21$.

${ }^{37}$ M. P. Allen and D. J. Tildesley, Computer Simulation of Liquids (Oxford University Press, 1989).

${ }^{38}$ M. Barisik and A. Beskok, Int. J. Therm. Sci. 77, 47 (2014).

${ }^{39}$ S. Plimpton, J. Chem. Phys. 117, 1 (1995).

${ }^{40}$ I.-C. Yeh and M. L. Berkowitz, J. Chem. Phys. 111, 3155 (1999).

${ }^{41}$ K. Binder, J. Horbach, W. Kob, W. Paul, and F. Varnik, J. Phys.: Condens. Matter 16, S429 (2004).

${ }^{42}$ F. Sofos, T. Karakasidis, and A. Liakopoulos, Int. J. Heat Mass Transfer 52, 735 (2009).

${ }^{43}$ C. T. Nguyen and B. Kim, Int. J. Precis. Eng. Manuf. 17, 503 (2016).

${ }^{44}$ D. Kim and E. Darve, Phys. Rev. E 73, 051203 (2006).

${ }^{45}$ R. Qiao and N. Aluru, Phys. Rev. Lett. 92, 198301 (2004).

${ }^{46}$ S. Wei, X. Xiaobin, Z. Hong, and X. Chuanxiang, Cryobiology 56, 93 (2008).

${ }^{47}$ I. Braslavsky and S. Lipson, Appl. Phys. Lett. 72, 264 (1998).

${ }^{48}$ P. Šunka, Phys. Plasmas 8, 2587 (2001).

${ }^{49}$ F. S. Cipcigan, V. P. Sokhan, A. P. Jones, J. Crain, and G. J. Martyna, Phys. Chem. Chem. Phys. 17, 8660 (2015).

${ }^{50}$ T. A. Ho and A. Striolo, Mol. Simul. 40, 1190 (2014).

${ }^{51}$ C. Y. Lee, J. A. McCammon, and P. Rossky, J. Chem. Phys. 80, 4448 (1984).

${ }^{52}$ M. Suk and N. Aluru, RSC Adv. 3, 9365 (2013).

${ }^{53}$ M. A. González and J. L. Abascal, J. Chem. Phys. 132, 096101 (2010).

${ }^{54}$ S. Tazi, A. Boțan, M. Salanne, V. Marry, P. Turq, and B. Rotenberg, J. Phys.: Condens. Matter 24, 284117 (2012). 
${ }^{55}$ G. S. Fanourgakis, J. Medina, and R. Prosmiti, J. Phys. Chem. A 116, 2564 (2012).

${ }^{56}$ W. Xiong, J. Z. Liu, M. Ma, Z. Xu, J. Sheridan, and Q. Zheng, Phys. Rev. E 84, 056329 (2011).

${ }^{57}$ P. Koumoutsakos, R. Jaffe, T. Werder, and J. Walther, Nanotechnology 2003(1), 148-151.

${ }^{58}$ W. R. Rice, Evolution 43, 223 (1989).

${ }^{59}$ N. Bjerrum, Science 115, 385 (1952).

${ }^{60}$ T. Hozumi, A. Saito, S. Okawa, and Y. Eshita, Int. J. Refrig. 28, 389 (2005).

${ }^{61}$ M. Orlowska, M. Havet, and A. Le-Bail, Food Res. Int. 42, 879 (2009).

${ }^{62}$ J. Wedekind, J. Wölk, D. Reguera, and R. Strey, J. Chem. Phys. 127, 154515 (2007).

${ }^{63}$ E.-M. Choi, Y.-H. Yoon, S. Lee, and H. Kang, Phys. Rev. Lett. 95, 085701 (2005).
${ }^{64}$ H. Qiu and W. Guo, Phys. Rev. Lett. 110, 195701 (2013).

${ }^{65}$ J. Yan and G. Patey, J. Chem. Phys. 139, 144501 (2013).

${ }^{66}$ J. Yan and G. Patey, J. Phys. Chem. A 116, 7057 (2012).

${ }^{67}$ D. R. Lide, CRC Handbook Chemistry and Physics (CRC Press, 2001-2002), p. 12J204.

$68 \AA$ Å. Melinder, Int. J. Refrig. 33, 1506 (2010).

${ }^{69}$ Y. Maréchal, The Hydrogen Bond and the Water Molecule: The Physics and Chemistry of Water, Aqueous and Bio-Media (Elsevier, 2006).

${ }^{70}$ J. Martí, J. Chem. Phys. 110, 6876 (1999).

${ }^{71}$ J. D. Smith, C. D. Cappa, K. R. Wilson, B. M. Messer, R. C. Cohen, and R. J. Saykally, Science 306, 851 (2004).

${ }^{72}$ K. Ritos, M. K. Borg, N. J. Mottram, and J. M. Reese, Philos. Trans. R. Soc., A 374, 20150025 (2016).

${ }^{73}$ V. F. Petrenko and R. W. Whitworth, Physics of Ice (OUP, Oxford, 1999). 